\title{
Receptor induced rearrangements of capsid and genome prime rhinovirus 14 for activation and uncoating
}

\author{
Dominik Hrebík, Tibor Füzik, Mária Gondová, Lenka Šmerdová, Athanassios Adamopoulos, Ondrej Šedo, Zbyněk \\ Zdráhal, Pavel Plevka
}

\author{
CEITEC MU, Brno, Czech Republic \\ dominik.hrebik@ceitec.muni.cz
}

Most of the rhinoviruses, which are the leading cause of common cold, utilize intercellular adhesion molecule-1 (ICAM-1) as a receptor to infect cells. Before genome release, rhinoviruses convert to activated particles that contain pores in the capsid, lack capsid proteins VP4, and have altered genome organization. The binding of rhinoviruses to ICAM-1 promotes virus activation; however, the molecular details of the process remain unknown. Here we present the structures of the native rhinovirus 14 and rhinovirus14-ICAM-1 complex at a resolution of 2.6 and $2.4 \AA$. The structures revealed a mechanism by which binding of rhinovirus 14 to ICAM-1 primes the virus for activation and subsequent genome release. The attachment of rhinovirus 14 to ICAM-1 induces conformational changes in the virion, which include translocation of the C-termini of VP4 subunits towards twofold symmetry axes of the capsid. Thus, VP4 subunits become poised for release through pores that open in the capsid upon particle activation. The cryo-EM reconstruction of rhinovirus 14 virion contains the resolved density of octa-nucleotides from the RNA genome, which interact with VP2 subunits near two-fold symmetry axes of the capsid. VP4 subunits with altered conformation, induced by the binding of rhinovirus 14 to ICAM-1, block the RNA-VP2 interactions and expose patches of positively charged residues around threefold symmetry axes of the capsid. The conformational changes of the capsid induce reorganization of the virus genome. The rearrangements of the capsid and genome probably lower the energy barrier of conversion of rhinovirus 14 virions to activated particles. The structure of rhinovirus 14 in complex with ICAM-1 represents an essential intermediate in the pathway of enterovirus genome release.

Keywords: rhinovirus, ICAM-1, cryo-EM, single particle analysis, receptor 